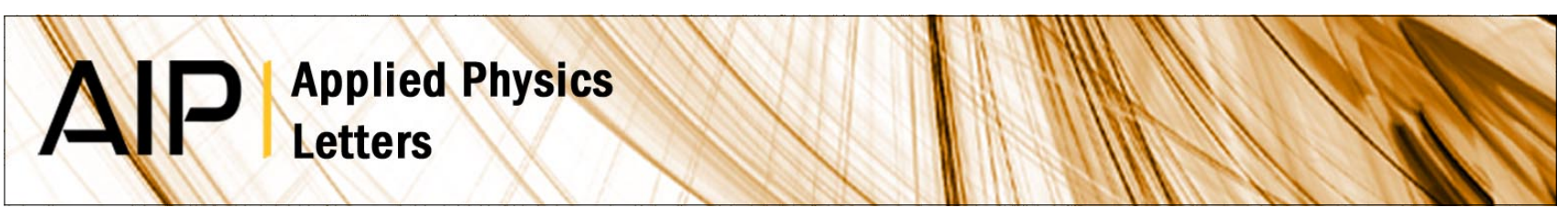

\title{
Backward elastic light scattering of malaria infected red blood cells
}

Seungjun Lee and Wei Lu

Citation: Appl. Phys. Lett. 99, 073704 (2011); doi: 10.1063/1.3627173

View online: http://dx.doi.org/10.1063/1.3627173

View Table of Contents: http://apl.aip.org/resource/1/APPLAB/v99/i7

Published by the AIP Publishing LLC.

Additional information on Appl. Phys. Lett.

Journal Homepage: http://apl.aip.org/

Journal Information: http://apl.aip.org/about/about_the_journal

Top downloads: http://apl.aip.org/features/most_downloaded

Information for Authors: http://apl.aip.org/authors

\section{ADVERTISEMENT}
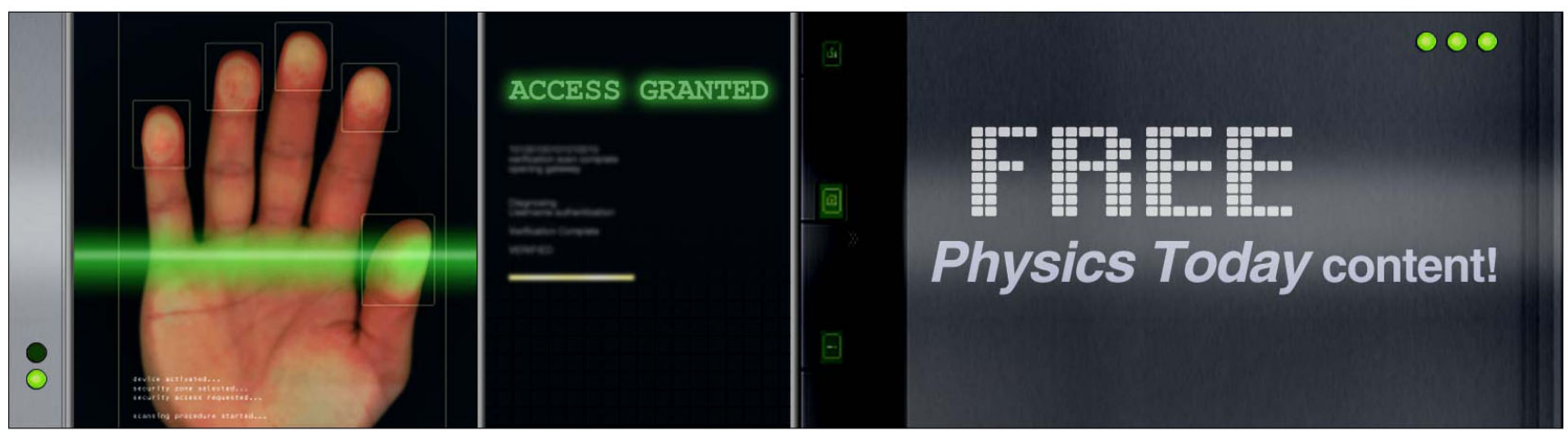


\title{
Backward elastic light scattering of malaria infected red blood cells
}

\author{
Seungjun Lee and Wei Lu ${ }^{\text {a) }}$ \\ Department of Mechanical Engineering, University of Michigan, Ann Arbor, Michigan 48109, USA
}

(Received 20 July 2011; accepted 2 August 2011; published online 19 August 2011)

\begin{abstract}
We investigated the backward light scattering pattern of healthy and malaria (Plasmodium falciparum) parasitized red blood cells. The spectrum could clearly distinguish between predominant ring stage infected blood cells and healthy blood cells. Further, we found that infected samples mixed with different stages of $P$. falciparum showed different signals, suggesting that even variance in parasite stages could also be detected by the spectrum. These results together with the backward scattering technique suggest the potential of non-invasive diagnosis of malaria through light scattering of blood cells near the surface of human body, such as using eyes or skin surface. (ㅇ 2011 American Institute of Physics. [doi:10.1063/1.3627173]
\end{abstract}

Malaria is a serious and fatal disease that causes 350500 million reported cases and nearly one million deaths annually. Malaria is currently diagnosed through microscopic examination of Giemsa-stained blood smears and parasite antigen or DNA-based tests. However, these methods require trained health workers, blood from patients, and a complex logistics system and laboratory infrastructure to process and test samples. An inherently non-invasive method would provide an innovative solution that can overcome the disadvantages of the current approaches. In this letter, we show that the signal from backward elastic light scattering can distinguish between healthy and malaria parasite Plasmodium falciparum invaded red blood cells.

When a particle scatters light upon illumination, the spatial distribution of the scattered light intensity is not random but forms a complex spatial pattern depending on the property of the particle. This process is a result of complicated interaction between the incident electromagnetic wave and the molecular or atomic structure of the scattering particle. When encountering a particle, the electromagnetic wave of an incident light beam periodically perturbs the electron orbits of the molecules or atoms in the particle. The induced oscillating dipole moments act as a source of electromagnetic radiation, giving off scattered light. Most of the light scattered by the particle is emitted at the same frequency of the incident light, which is known as elastic scattering. Elastic scattering can be used to distinguish particles of different structure or material characteristics, such as size, morphology, and chemical composition. The principle is applicable to biological systems to discriminate between different cell types or states. Studies have shown that elastic light scattering can be used for the detection of breast cancer ${ }^{1}$ or monitor the biological process that accompanies biochemical and morphological changes. ${ }^{2}$

Light scattering of red blood cells has been studied through both experiments ${ }^{3-5}$ and numerical simulations. ${ }^{6-8}$ Recently this technique has been extended to study malaria parasite invaded red blood cells, including UV-visible light spectroscopy of different stages of infected red blood cells with the zero forward direction of the incident light ${ }^{9}$ and light

\footnotetext{
${ }^{\text {a) }}$ Author to whom correspondence should be addressed. Electronic mail: weilu@umich.edu.
}

scattering of an individual red blood cell. ${ }^{10}$ Previous attempts on cell scattering have been focusing on diluted samples and forward scattering. Sample preparation and the requirement to place the light source and detector on opposite sides of the sample have fundamentally limited the techniques to a lab environment and invasive blood drawing. In this letter we focus on undiluted samples and backward scattering, where the light source and detector are on the same side. This setup allows potentially detecting scattering of malaria infected red blood cells from eyes or skin surfaces for in vivo diagnosis. The red blood cells invaded by the $P$. falciparum malaria parasite experience significant modification in the structural, mechanical, and biochemical properties, ${ }^{10-14}$ including morphological deviation from the biconcave shape, reduction of membrane fluctuations and elasticity, and decrease of hemoglobin concentration. We envision that backward elastic light scattering is capable of detecting these changes and showing spectrum difference from that of healthy cells.

The optical setup is shown in Fig. 1, which consists of a lamp, a spectrometer, a lens, optic fibers, and a variable aperture. The optic components are aligned vertically so that a flat container with liquid samples, such as a glass slide, can be placed horizontally. A broadband light source is delivered through a $400 \mu \mathrm{m}$ optic fiber and focused on a small test spot by a plano-convex lens with a focal length of $\mathrm{f}=25.4 \mathrm{~mm}$. The blood sample is placed at the waist of the light beam on top of a glass slide. The distance between the sample and the lens is about $70 \mathrm{~mm}$. A collecting fiber which collects the scattered light is attached to a computer-controlled motorized rotation stage with a 5 arc min resolution. This stage allows precise control and change of the orientation of the collecting fiber relative to the incident beam so that the scattered light from the test spot can be measured at various angles, $\theta$. The collecting fiber passes the scatted light at each angle to a spectrometer that is connected to a computer via USB. A variable aperture is used to control the diameter and intensity of the light. The aperture is adjusted so that the light passing through the sample does not directly get into the collecting fiber and interference with the scattered light.

The scattering intensity was calculated by $I_{\text {particle }}(\theta)=\left(I_{\text {sample }}(\theta)-I_{\text {backgraound }}(\theta)\right) / I_{\text {reference. }}$. $I_{\text {reference }}$ is the intensity measured with the collecting fiber aligned at 


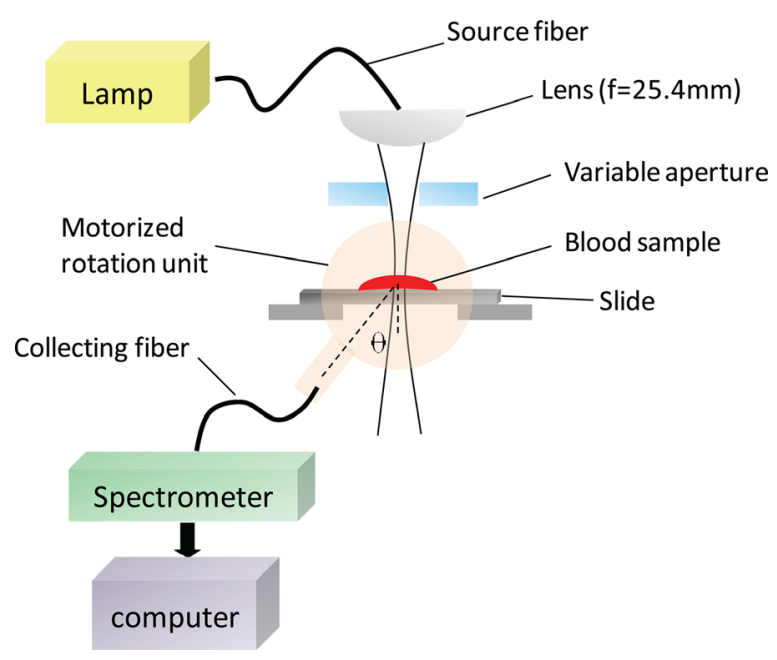

FIG. 1. (Color online) Schematic of the optical setup for light scattering measurement.

zero degree $(\theta=0)$, which gives the spectrum of the light source. $I_{\text {background }}(\theta)$ is measured at each angle with light passing through the glass slide without any sample on top of it. $I_{\text {sample }}(\theta)$ is obtained with the sample on top of the glass slide. Subtracting $I_{\text {backgraound }}(\theta)$ removes any effect of the glass slide. The calculated intensity for each angle is further normalized by the area under the corresponding intensity curve to remove the effect of the number of particles in the test spot. With this algorithm, we found that the beam diameter and location had no influence on the spectrum. A larger beam diameter gave a stronger signal, allowing a shorter integration time.

Preparation of the healthy and parasitized red blood cell samples were conducted at the Malaria Branch in Centers for Disease Control and Prevention (CDC). The infected blood was obtained from the field station in Kenya, and cultured at the CDC Lab for different malaria stages (rings, trophozoites, schizont). Briefly, the laboratory-adapted W2 or 3D7 strains of $P$. falciparum were used in this study. The bloodstage parasites were cultured in RPMI 1640 medium supplemented with $10 \%$ heat inactivated O+human serum, $1 \mathrm{mg} / \mathrm{ml}$ of gentamicin, $36 \mathrm{mM}$ hypoxanthine, $31 \mathrm{mM}$ HEPES, and $25 \mathrm{mM}$ sodium bicarbonate. The parasite cultures were adjusted to either $2 \%$ parasitemia with predominant ring stage parasite or varied \% parasitemia with different stages of parasites in 50\% hematocrit. The healthy red blood cells in 50\% hematocrit were used as a negative control. The samples with the $50 \%$ hematocrit and the samples further diluted with phosphate buffered saline (PBS) were used for testing.

Each sample was measured three times, and the results were averaged. In fact, the three measurements gave almost identical results. We have performed both forward $\left(\theta<90^{\circ}\right)$ and backward scattering $\left(\theta>90^{\circ}\right)$ with and without sample dilution. Our studies showed that healthy blood samples demonstrated the same signature spectrum for a given detecting angle. We also found that while the spectrum curve depended on the detecting angle, it was not sensitive to small angle changes for undiluted samples. The backscattering results are presented below.

Malaria parasites grow following the ring, trophozoite, and schizont stages. The early predominant ring stage parasites are common in human peripheral blood. Figure 2 shows
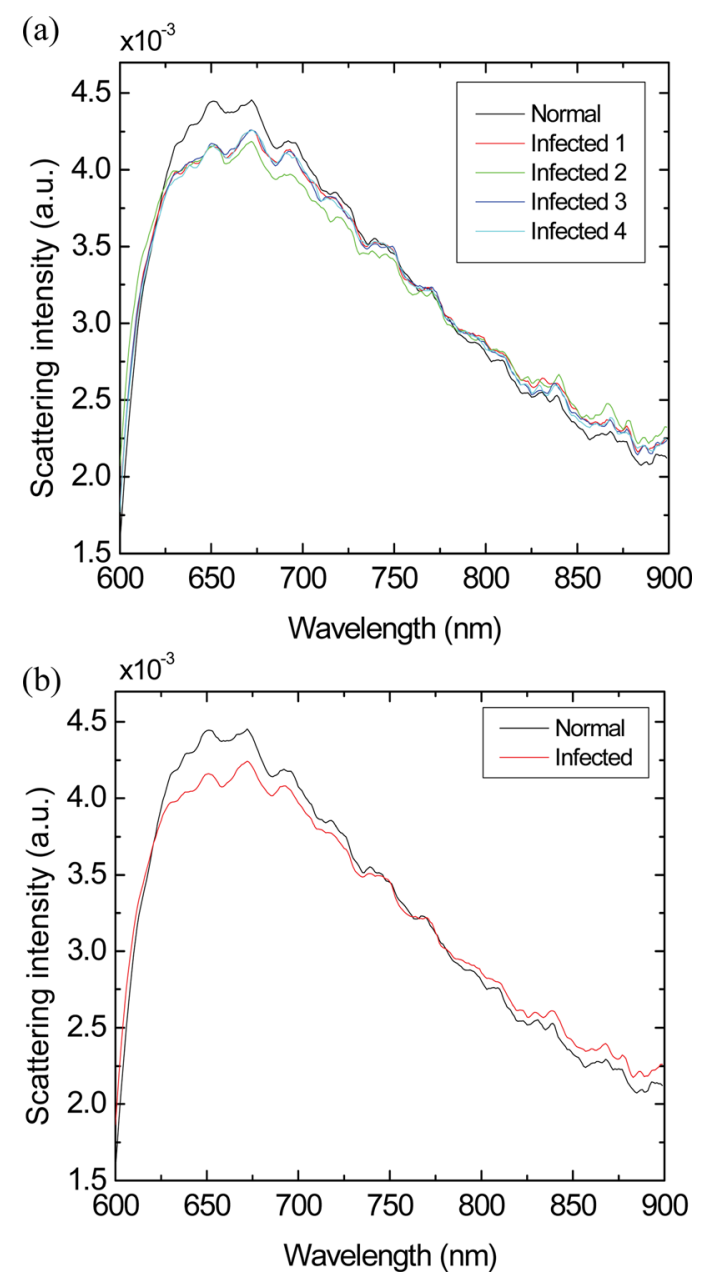

FIG. 2. (Color online) Backward scattering of healthy and infected predominant ring stage samples, measured at $\theta=150^{\circ}\left(30^{\circ}\right.$ from the opposite incident beam direction): (a) comparison of healthy and four infected samples in Table I and (b) comparison of healthy and the average of the infected samples in (a).

an example of our study. The data of the samples are given in Table I. The second column shows the percentage of red blood cells that are infected among all red blood cells in each sample. The next three columns show the distribution of parasite stages in the infected red blood cells. Most of the samples are predominant ring stage except No. 2. A clear difference is observed between the spectra of the healthy and infected samples, as shown in Fig. 2(a). The curves of the infected samples are close to each other except the green one, which corresponds to sample No. 2. This sample has $50 \%$ late stage schizont and shows a larger spectrum difference from that of the healthy sample. Figure 2(b) compares the average of the four infected samples to the healthy sample. The spectrum intensity of the infected samples is weaker

TABLE I. Data of predominant ring stage samples.

\begin{tabular}{lccccc}
\hline \hline $\begin{array}{c}\text { Sample } \\
\text { No. }\end{array}$ & $\begin{array}{c}\text { Total } \\
(\%)\end{array}$ & $\begin{array}{c}\text { Rings } \\
(\%)\end{array}$ & $\begin{array}{c}\text { Trophozoites } \\
(\%)\end{array}$ & $\begin{array}{c}\text { Schizont } \\
(\%)\end{array}$ & $\begin{array}{c}\text { Hematocrite } \\
(\%)\end{array}$ \\
\hline 1 & 3.5 & 94.2 & 0.0 & 5.8 & 50 \\
2 & 2.0 & 50.0 & 0.0 & 50.0 & 50 \\
3 & 3.2 & 81.2 & 6.2 & 12.6 & 50 \\
4 & 3.8 & 84.2 & 0.0 & 15.8 & 50 \\
\hline \hline
\end{tabular}


TABLE II. Data of infected samples with different $\%$ parasitemia and stages.

\begin{tabular}{lccccc}
\hline \hline $\begin{array}{c}\text { Sample } \\
\text { No. }\end{array}$ & $\begin{array}{c}\text { Total } \\
(\%)\end{array}$ & $\begin{array}{c}\text { Rings } \\
(\%)\end{array}$ & $\begin{array}{c}\text { Trophozoites } \\
(\%)\end{array}$ & $\begin{array}{c}\text { Schizont } \\
(\%)\end{array}$ & $\begin{array}{c}\text { Hematocrite } \\
(\%)\end{array}$ \\
\hline 1 & 1.9 & 58.0 & 10.5 & 31.5 & 50 \\
2 & 2.1 & 0.0 & 19.0 & 81.0 & 50 \\
3 & 3.2 & 28.2 & 9.3 & 62.5 & 50 \\
4 & 2.2 & 9.0 & 36.5 & 54.5 & 50 \\
\hline \hline
\end{tabular}

in the window of $620-750 \mathrm{~nm}$ but is stronger beyond 750 $\mathrm{nm}$. We measured different samples at several scattering angles up to $\theta=163^{\circ}$ and found that the crossover at about $750 \mathrm{~nm}$ appeared to be general. We have not tested larger scattering angles because of interference between the rotational arm and the lens in our current setup.

We tested infected samples mixed with different stages of $P$. falciparum to examine the influence of variance in parasite stages on the spectrum. The data about the samples and their mixed stages are listed in Table II. The results of backscattering measurement are shown in Fig. 3. Comparing with Fig. 2(a), we can observe more variance between the curves of the infected samples in Fig. 3(a), and they are all clearly
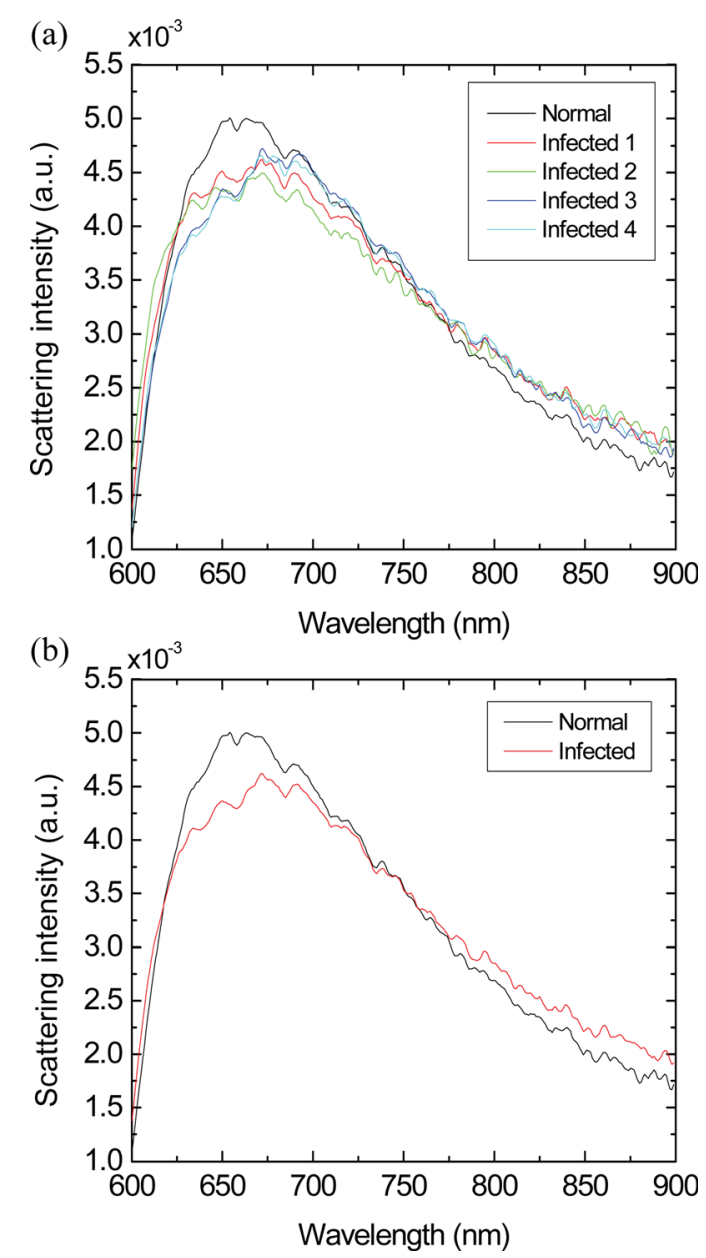

FIG. 3. (Color online) Backward scattering of healthy and infected samples, measured at $\theta=150^{\circ}$ ( $30^{\circ}$ from the opposite incident beam direction): (a) comparison of healthy and infected samples at different stage mixture in Table II and (b) comparison of healthy and the average of the infected samples in (a). distinguishable from the spectrum of the healthy sample. The curve of the No. 2 sample, which contains more schizont stage, shows the largest difference from the healthy sample. This suggests that the spectrum difference increases as the stage of infection progresses. Figure 3(b) compares the spectra of the healthy and the average of infected samples. Overall, the trend is similar to Fig. 2. The spectrum intensity of the infected samples is weaker than that of the healthy sample below $750 \mathrm{~nm}$ and stronger above $750 \mathrm{~nm}$. Figure 3(b) shows a larger difference from the healthy sample than that in Fig. 2(b) because it has more predominant schizont stage; on the contrary, the average in Fig. 2(b) is predominant ring stage.

In conclusion, we report that healthy and malariainfected red blood cells show a clear spectrum difference in elastic light scattering. The results of backward scattering on undiluted samples suggest elastic light scattering as a potential tool for non-invasive diagnosis. For instance, it may be possible to aim a light beam at blood vessels in the iris or retina and capture the scattered signal. Malaria parasites consume hemoglobin and produce digestive waste, hemozoin crystal, in infected red blood cells. There is significant difference of material properties between hemozoin, the compositions of malaria parasites, and the normal contents of human cells. We think this process causes significant structural and compositional changes of the cells, leading to a signature change of the light scattering pattern. This work is a first step. Development of practical non-invasive diagnosis needs much more further studies, such as investigating the spectrum of other malaria parasite species though $P$. falciparum which is most dangerous or identifying the effect of thin blood vessels on scattering signals.

The authors acknowledge financial support by a grant from the Bill \& Melinda Gates Foundation through the Grand Challenges Explorations Initiative. We thank Dr. Ya Ping Shi at the Malaria Branch, Centers for Disease Control and Prevention, Atlanta, Georgia for the preparation of the samples.

${ }^{1}$ I. J. Bigio, S. G. Bown, G. Briggs, C. Kelley, S. Lakhani, D. Pickard, P. M. Ripley, I. G. Rose, and C. Saunders, J. Biomed. Opt. 5, 221 (2000).

${ }^{2}$ C. S. Mulvery, A. L. Curtis, S. K. Singh, and I. J. Bigio, IEEE J. Sel. Top. Quantum Electron. 13, 1663 (2007).

${ }^{3}$ J. M. Steinke and A. P. Shepherd, Appl. Opt. 27, 4027 (1988).

${ }^{4}$ M. Hammer, D. Schweitzer, B. Michel, E. Thamm, and A. Kolb, Appl. Opt. 37, 7410 (1998).

${ }^{5}$ D. H. Tycko, M. H. Metz, E. A. Epstein, and A. Grinbaum, Appl. Opt. 24, 1355 (1985)

${ }^{6}$ J. He, A. Karlsson, J. Swartling, and S. Andersson-Engels, J. Opt. Soc. Am. A 21, 1953 (2004).

${ }^{7}$ S. V. Tsinopoulos, E. J. Sellountos, and D. Polyzos, Appl. Opt. 41, 1408 (2002).

${ }^{8}$ A. M. K. Nilsson, P. Alsholm, A. Karlsson, and S. Andersson-Engels, Appl. Opt. 37, 2735 (1998).

${ }^{9}$ Y. M. Serebrennikova, J. Patel, and L. H. Garcia-Rubio, Appl. Opt. 49, 180 (2010).

${ }^{10}$ Y. Park, M. Diez-Silva, D. Fu, G. Popescu, W. Choi, I. Barman, S. Suresh, and M. S. Feld, J. Biomed. Opt. 15, 020506 (2010).

${ }^{11}$ D. E. Goldberg, A. F. G. Slater, A. Cerami, and G. B. Henderson, Proc. Natl. Acad. Sci. U.S.A. 87, 2931 (1990).

${ }^{12}$ Y. K. Park, M. Diez-Silva, G. Popescu, G. Lykotrafitis, W. Choi, M. S. Feld, and S. Sutresh, Proc. Natl. Acad. Sci. U.S.A. 105, 13730 (2008).

${ }^{13}$ S. Suresh, J. Spatz, J. P. Mills, A. Micoulet, M. Dao, C. T. Lim, M. Beil, and T. Seufferlein, Acta Biomater. 1, 15 (2005).

${ }^{14}$ B. M. Cooke, N. Mohandas, and R. L. Coppel, Adv. Parasitol. 50, 1 (2001). 